\title{
Development of a High Altitude LAGO Site in Peru
}

\section{Otiniano ${ }^{1}$, S Vargas ${ }^{* 2}$, F Quispe ${ }^{1}$, W Guevara ${ }^{1}$, For The LAGO Collaboration ${ }^{3}$}

${ }^{1}$ Dirección de Astrofísica Comisión Nacional De Investigación y Desarrollo Aeroespacial Lima, Perú

${ }^{2}$ Escuela Politécnica Nacional

Quito, Ecuador

${ }^{3}$ lagoproject.org, see the full list of members and institutions at lagoproject.org/collab.html

E-mail: lotiniano@conida.gob.pe

The Latin American Giant Observatory (LAGO) Project is an extended Cosmic Ray Observatory mainly oriented to perform basic research in three branches: high energy phenomena, space weather and atmospheric radiation at ground level. To observe the high energy component (over $10 \mathrm{GeV}$ ) of Gamma Ray Bursts (GRBs), the LAGO Collaboration is installing Water Cherenkov Detectors ) in high altitude sites.

Extensive Air Showers produced in the atmosphere by Gamma Ray Bursts high energy photons could be detected by WCD arrays given their good sensitivity to secondary photons and other particles in the cascades, by looking for excesses over the secondary particle flux. In this work the current developments to build and characterize a high altitude (>4600 m a.s.1.) LAGO site at the central highlands of Peru are described.

The 34th International Cosmic Ray Conference,

30 July- 6 August, 2015

The Hague, The Netherlands

${ }^{*}$ Speaker. 


\section{Introduction}

One of the LAGO international collaboration goals is to observe high energy component (over $10 \mathrm{GeV}$ ) of Gamma Ray Bursts (GRBs) in arrays of Water Cherenkov Detectors (WCD) at high mountain sites [6], using the single particle technique [7]. When a GRB occurs, the atmosphere is impacted by a multitude of high energy gamma rays that produces decay showers. Together those showers could be detectable at ground level as an instant excess in the flux measured by a detector. Showers initiated by gamma rays have a composition of $90 \%$ photons, $9 \%$ electrons and $<1 \%$ muons, WCDs are sensitive to all those particles. These showers are absorbed high in the atmosphere, not being able to survive and reach the ground. In order to detect the greatest possible number of particles, the WCDs must be placed at high altitude.

In Peru a new array of the WCDs for the LAGO project is under development. In this article we describe the site chosen for this purpose and the detector prototype, then we describe a GEANT4[3] simulation of the detector and his validation using data of the prototype. Finally we discuss the perspectives for the installation at high altitude of the array at the top of Huaytapallana's mountain at 4800 m.a.s.l.

\section{Huancayo Site and Experimental Setup}

The Huayao Magnetic Observatory $(\mathrm{OMH})$ is located in the Central Sierra of Peru (12"02.7' N $75^{\prime \prime} 20.4^{\prime} \mathrm{W}$ ) on a vast plain at 3300 m.a.s.1.. Since 1936 it has hosted secondary cosmic rays ground experiments (a Forbush Sphere and Neutron Monitor), currently makes geomagnetic and meteorological records, monitoring of solar activity and has a seismic station[2]. For monitoring detectors and storage of materials a mobile station has been built using steel containers.

The LAGO WCD is commonly made of a water commercial tank, filled with filtered and chemically purified water, a external coberture for light isolation and an internal liner of Tyvek, to reflect and diffuse Cherenkov photons produced in water by high energy secondary cosmic rays. The diffusion of Cherenkov photons reduces the signal dependence with the secondary particle trajectory within the detector. A large area photomultiplier PMT complete the detector[1]. Our previous experience in high altitude deploy of WCDs in Peru [10] has shown the need to minimize the size and weight of the detectors components in order to facilitate and optimize cost of deployment. Thus we developed a prototype made of a cylindrical surface ( $3 \mathrm{~m}$ diameter $1 \mathrm{~m}$ height) assembled using four stainless steel plates that support two cylindrical bags filled with treated water, the external bag, made of high density polyethylene, provides light isolation, the internal bag is made of Tyvek. All the detector is also covered with an external coating of plastic blanket for environmental and also light isolation. The detector is completed with an upper 9354KB Electron Tube (PMT), see Figure 3.

The typical signal produced by the detector being crossed by a particle is a negative pulse with a fast decay and an exponential recovery, the pulse amplitude is $500 \mathrm{mV}$ and width of $150 \mathrm{~ns}$. The LAGO project has development its own DAQ system with three channels at $40 \mathrm{MHz}$ sampling rate 10 bits digitalization (ADC unit $\approx 1 \mathrm{mV}$ ), time stamp via GPS signal, sampling of pressure and temperature each second and control for high voltage powers supply needed to operate and record the signal of PMTs[1]. 

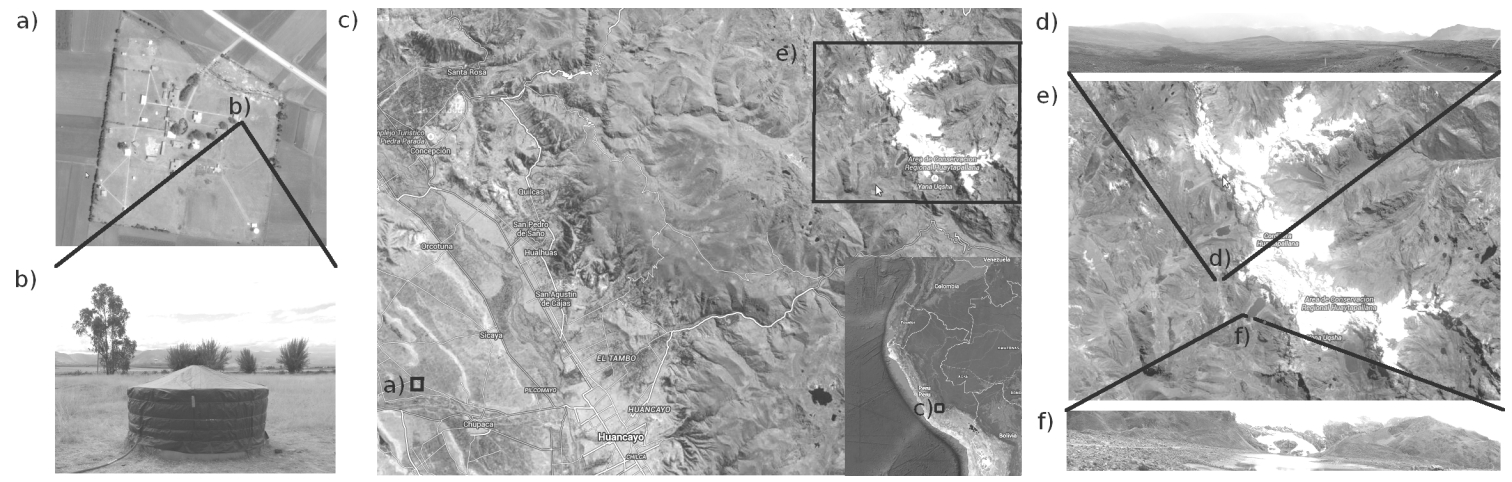

Figure 1: Huancayo site location: $30 \mathrm{~km}$ from the city of Huancayo (c) the Huayao Magnetic Observatory (OHM) (a) houses our WCD prototype (b). Nearby there are two sites competing in the Huaytapallana mountain to accommodate an array of detectors. One at. 4600 m.a.s.l. having a nearby lagoon (f), the other at 4800 m.a.s.l. with no nearby water sources, but with easy access by road (d).

\section{Detector Background Simulation Validation}

The LAGO collaboration has developed CORSIKA based tools to simulate radiation environments of secondary cosmic rays[8]. Corrected for geomagnetic effects by cutting the spectrum of primaries reaching the atmosphere. A campaign for simulations of all sites of LAGO with WCDs is on development. Figure 2 shows the result of simulation spectrum of secondary cosmic rays for the $\mathrm{OMH}$ at 3300 m.a.s.1. and for the same coordinates but at 4800 m.a.s.l.

In order to calibrate the response of WCDs to this radiation environments, a GEANT4 detector simulation has been developed. This simulation must be versatile enough to include all models of WCDs used in the collaboration, which vary principally because of the local materials used in manufacturing on each site to reduce costs. So different geometries due to the use of water tanks acquired in each country, different types of PMTs and different water treatments should be taken into account [11].

The simulation uses a input table that allows to vary the geometry of the cylindrical water tank, the size and type (by varing the quantum efficiency model) of the PMT and the mean free path of photons in water (a percent of the mean free path in pure water, $0<\alpha<1$ parameter). Also a model of the tyvek reflection is implemented based on [5] with a total reflection parameter implemented $(0<\beta<1)$.

In order to calibrate the WCD we tune up unknown parameters ( $\alpha$ and $\beta$ ) to adjust the pulse shape signal response of the prototype WCD measured with the DAQ produced simulated vertical muons reaching the detector from top. Then we simulate the response of the detector to the secondary cosmic rays generated using CORSIKA simulation toolkit [8]. A comparison between these simulations and the spectrum measured with the detector of the OMH prototype is shown in Figure 3, we can notice that we are missing the low energy spectrum of gammas that reach the detector, this is probably due by the high level of trigger we are using for the pulses, 100 ADC units $(\approx 100 \mathrm{mV})$ to avoid noise. 


\section{Discussion and Conclusions}

We are planning the installation of an array of WCD at 4600 or 4800 m.a.s.l. in central Andes of Peru, for the LAGO project. In the coming months two more detectors will be added to form a triangular array in order to test coinciding acquisition mode.

The detector is in process of characterization using simulations and we have developed a protocol and a series of logistics process to easily install it. However, it is necessary to refine the simulation, in particular identifying the Vertical Equivalent Muon ${ }^{1}$ (VEM ) on the measured load spectrum in the WCD pulses signal. Besides, it is necessary to amplify the signal to observe the electromagnetic spectrum at low energies, the simulation can be used to study two strategies to do that: adding a wavelength shifter in the water of the detector to increase the number of photons detected or including an electronic preamp. We also hope that the simulator allows us to estimate the response to the flux of high energy gamma generated at ground level by GRBs.

\section{Acknowledgments}

The autors gratefully acknowledges the financial support from Fondo para la Innovación, Ciencia y Tecnología (FINCyT) trough the project PIBA-2-P-020-14. The LAGO Collaboration is very thankful to the Pierre Auger Collaboration for its continuous support.

\section{References}

[1] H. Asorey for the LAGO Collaboration. LAGO: the latin american giant observatory. This Procedings, volume in press, 2015.

[2] J. Bravo. El Aporte científico del Observatorio de Huancayo. ET DOCTORES, MAGJSTRI., v 11, 4-8, 1995.

[3] S. Agostinelli, J. Allison, K. Amako, J Apostolakis, H. Araujo, P. Arce, M. Asai, D Axen, S. Banerjee, G. Barrand, et al. Geant4: a simulation toolkit. Nuclear instruments and methods in physics research section A: Accelerators, Spectrometers, Detectors and Associated Equipment, 506(3):250-303, 2003.

[4] D. Heck et al. CORSIKA : A Monte Carlo Code to Simulate Extensive Air Showers. FZKA,6019:1-98, 1998.

[5] A. Chevarria. A study on the reflective properties of Tyvek in air and underwater (Senior Thesis). USA: the Department of Physics of Trinity College, Duke University, 2007.

[6] D. Allard, I. Allekotte, C. Alvarez, H. Asorey, et al., Use of water-Cherenkov detectors to detect Gamma Ray Bursts at the Large Aperture GRB Observatory (LAGO), Nuclear Instruments and Methods in Physics Research Section A: Accelerators, Spectrometers, Detectors and Associated Equipment 595 (1),70âĂŞ72, 2008

[7] M. Aglietta, et al., Search for Gamma-Ray Bursts at Photon Energies E $>=10 \mathrm{GeV}$ and E $>=80 \mathrm{TeV}$. Astrophysical Journal v.469, p.305, 1996

\footnotetext{
${ }^{1}$ Signal produced by vertical muons that cross the WCDs. The angular distribution of secondary cosmic rays arriving at ground has its maximum in vertical, so this is reflected in the measured flow of the detector, see[12].
} 

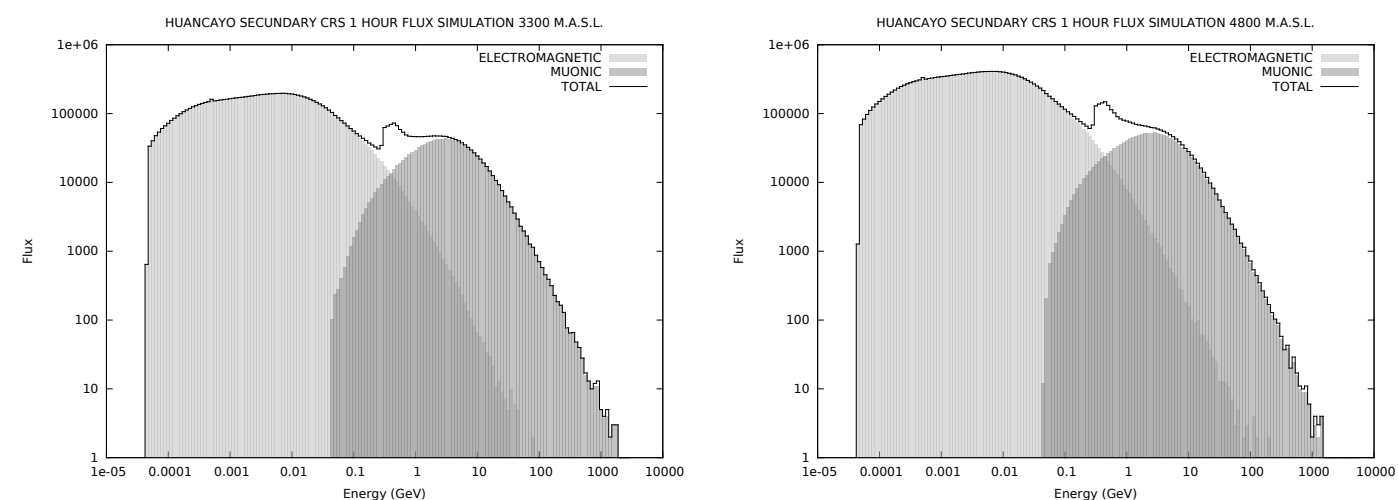

Figure 2: Simulated secondary cosmic ray spectrum in the location of the $\mathrm{OMH}$ at $3300 \mathrm{~m}$.a.s.l. (left) and 4800 m.a.s.l. (right). In both we show the main components of the flux (electromagnetic and muon). Note also the expected increased electromagnetic component at higher altitude.
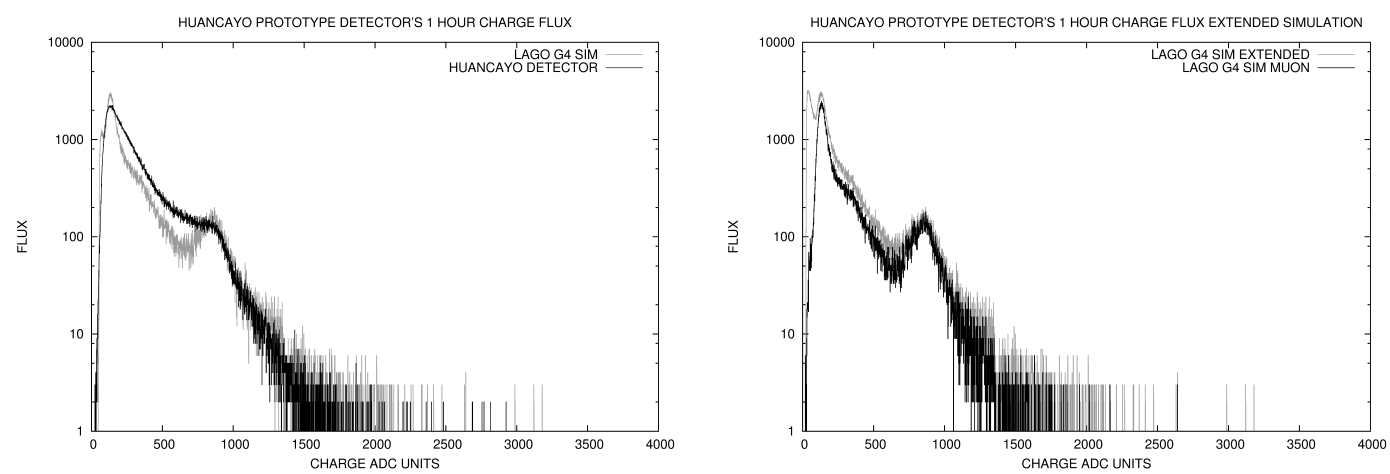

Figure 3: Simulated and measured charge spectra for the prototype WCD in the OMH. Left: Comparison of the spectra. Right: simulated muon spectrum component and the spectrum expected with no trigger level.

[8] M.S. Durán, H. Asorey, S. Dasso, L. Nuñez, Y. Peréz, C. Sarmiento for the LAGO Collaboration. The LAGO space weather program: Directional geomagnetic effects, background fluence calculations and multi-spectral data analysis. This Procedings, volume in press, 2015.

[9] C. Sarmiento, L.A. Nuñez-Castiñeyra, H. Asorey, L. Nuñez, P. Miranda, C. Nina, C. Salinas and R. Ticona for the LAGO Collaboration Analysis of background cosmic ray rate in the 2010-2012 period from the LAGO detectors at Chacaltaya. This Procedings, volume in press, 2015.

[10] Tueros-Cuadros, et al. The LAGO (Large Aperture GRB Observatory) in Peru. Proceedings of the International Astronomical Union volume 7(S286), 445-447,2011

[11] I. Sidelnik for the LAGO Collaboration The Sites of the Latin American Giant Observatory, This Procedings, volume in press, 2015.

[12] J. Ridky, et al. The surface detector of the Pierre Auger Observatory. Nuclear Physics B (Proc. Suppl.), 2007. 